\title{
¿LA DESIGUALDAD DE LOS AGENTES AFECTA AL CONSUMO? UNA VISIÓN DESDE LA POLITTICA MONETARIA
}

\section{RESUMEN}

El presente documento analiza el efecto indirecto entre consumo agregado y el diseño de política monetaria bajo el enfoque neokeynesiano básico en una economía pequeña y cerrada. Asumiendo agentes heterogéneos, se observa que el impacto de la tasa de interés sobre el consumo es mayor que considerar un modelo de agente representativo según Kaplan, Moll y Violante (2018). Así, el trabajo de investigación demuestra que si el gobierno genera una política monetaria que incentive a la economía, su impacto es mayor en aquellas familias que poseen un portafolio diversificado cuya riqueza está dividida entre salarios y activos financieros, que en aquellas que solamente cuentan con ingresos laborales, ampliando la brecha de desigualdad.

JEL: E12; E21; E42; E52; D63

PALABRAS CLAVES: Agentes heterogéneos; consumo; política monetaria, desigualdad.

\begin{abstract}
This document analyzes indirect effect between aggregate consumption and monetary policy design in the basic new keynesian model in small-close economies. Assuming heterogeneous agents, the impact of interest rate over consumption is bigger than considering a representative agent model according to Kaplan, Moll and Violante (2018). So, this working paper prove if the government generates a monetary policy design that incentives to economy, the impact will be more effects into families with diversified portfolio whose wealth is divided by salaries and financial assets, than whom only having working incomes, expanding noticeable the inequality gap.
\end{abstract}

JEL: E12; E21; E42; E52; D63

KEYWORDS: Heterogeneous agents; consumption; monetary policy, inequality.

A Investigador docente de la Escuela Profesional de Economía de la Facultad de Ciencias Contables, Económicas y Financieras de la Universidad de San Martín de Porres. 


\section{INTRODUCCIÓN}

El consumo agregado es uno de los componentes más importantes del Producto Bruto Interno (PBI), de allí que resulta fundamental entender cómo la política monetaria afecta a este importante agregado en el tiempo. Uno de los principales mecanismos que se destaca en la aplicación de un modelo neokeynesiano básico es el de la transmisión monetaria que ocurre a través de la sustitución intertemporal del consumo. Sin embargo, la evidencia empírica muestra que este canal opera únicamente para una pequeña masa de consumidores, aquellos que tienen acceso al sistema financiero y mantienen la mayor parte de su riqueza como depósitos o activos líquidos de corto plazo. Esta proporción es aún más pequeña en economías de ingresos medios y bajos, en donde la participación en el sistema financiero es aún menor.

Entonces, ¿cómo afecta la política monetaria al consumo agregado y al PBI en este tipo de economías? ¿qué tipo de mecanismos de transmisión son los más importantes: directos o indirectos?, es decir, ¿es el efecto de la política monetaria sobre los ingresos laborales o sobre el mercado de activos el más importante para explicar la dinámica del consumo agregado? Este trabajo responde estas preguntas utilizando un modelo de agentes heterogéneos para una economía cerrada, en donde la política monetaria tiene efectos reales. Cabe señalar que en economías emergentes existe una marcada división de la población en dos tipos de familias (ver Tabla 1). El primero tiene la posibilidad de contar con activos tras un mayor acceso a mercados financieros; mientras que el segundo sólo cuenta con sus ingresos salariales, es decir dependen únicamente de su fuerza laboral.

\section{Tabla 1}

Como lo menciona Aiyagari (1994), no contar con ingresos diversificados puede tener consecuencias para aquellas familias cuyo consumo sólo dependa de su nivel de ingreso laboral, no pudiendo optimizar intertemporalmente tras no tener ahorros preventivos que permitan amortiguar cualquier choque idiosincrático. Por lo tanto, experimentan fuertes volatilidades en los niveles de consumo. Así, en este aspecto la política monetaria se muestra poco efectiva ya que los cambios en las tasas de interés no podrían reducir dichas volatilidades.
Los recientes trabajos demodelos neokeynesianos como el de McKey y Reis (2016); Auclert (2017); Galí (2018); Kaplan et al (2018); entre otros, permiten capturar la heterogeneidad de los ingresos. Muestran que la respuesta (directa) del consumo ante cambios en la tasa de interés es mucho más baja comparado con modelos de agente representativo, es decir un 33\% frente a un $95 \%$ de respuesta. Sin embargo, la figura es opuesta al observar la respuesta (indirecta) ante cambios en el ingreso laboral, el cual es mucho más alta, mostrando un $66 \%$ frente a un $5 \%$, respectivamente.

Así, el presente trabajo tiene como principal objetivo mostrar la existencia de una baja sensibilidad del consumo agregado respecto a cambios en la tasa de interés en economías pequeñas y abiertas; opuestas al que se tiene ante cambios en los ingresos laborales. Para ello, se utiliza un modelo neokeynesiano bajo el enfoque propuesto por Clarida, Galí, Gertler (1999); y Woodford (2003), aplicando algoritmos de simulación à-la Bewley presentados en los trabajos de Huggett (1993) y Aiyagari (1994) a $\mathrm{n}$ de diagnosticar cuáles son los efectos de la política monetaria sobre el consumo en este tipo de economías.

Por ejemplo en el caso peruano, entre el 2008 y 2018 los estratos sociales menos favorecidos presentan Propensiones Marginales a Consumir $(\mathrm{PMgC}) 1$ diferentes a la media de quienes pueden suavizar su consumo ante períodos de alta incertidumbre en la economía2 (ver Figura 1). Así, en el estrato social con menos ingresos 3 ( $1^{\circ}$ decil y quintil) y donde existe un subgrupo de personas cuyos ingresos muestran variaciones muy pequeñas, su $\mathrm{PMgC}$ promedio es 0.77 ; cifra mayor a 0.69 de aquellos con mayor tasa de variación. Lo resaltante en los

\footnotetext{
$1 \mathrm{La} \mathrm{PMgC} \mathrm{se} \mathrm{construye} \mathrm{recogiendo} \mathrm{datos} \mathrm{de} \mathrm{gasto} \mathrm{de} \mathrm{consumo} \mathrm{y}$ de ingreso cuya base está compuesta por gastos en alimentos; vestido y calzado; alquiler de vivienda; combustible; electricidad; muebles y enseres; conservación y mantenimiento de hogares; equipos; cuidados; conservación de la salud; servicios médicos; transporte y comunicaciones; esparcimiento; diversión; servicios culturales y de enseñanza; entre otros. Y por el lado del ingreso, compuesto por ingresos laborales; tanto monetario como de bienes; por actividades principales o secundarias, dependientes o independientes; ingresos extraordinarios; transferencias monetarias del pais o del extranjero, sean privadas o públicas; alquiler o transferencia de vivienda; entre otros, cuya fuente es la Encuesta Nacional de Hogares del INEI.

2 Según Kaplan et al (2018) señala que en los modelos con agente representativo se asume un consumidor con ingreso permanente y quien no responde a cambios transitorios en su ingreso. En línea, se encuentra la evidencia cuasiexperimental desarrollada por Parker et al. (2014) que muestra que la PMgC agregada se mantiene sin grandes variaciones en el largo plazo.

3 De acuerdo al Banco Mundial (2018) la población peruana tiene como Coeficiente de Gini cerca de $44 \%$, siendo uno de los países menos desiguales entre los países con mayor PBI de la región.
} 
datos es que la PMgC es bastante menor en este último considerándose que tienen una mayor disposición a ahorrar ante mayores recursos. Mientras que en el estrato social con mayores ingresos $\left(10^{\circ}\right.$ percentil y $5^{\circ}$ quintil), aquel subgrupo con variaciones muy pequeñas en su ingreso muestran un $\mathrm{PMgC}$ promedio de 0.76; y 0.88 para aquellos con mayor variación. Ello refuerza la hipótesis de que la heterogeneidad en el ingreso impacta en el consumo de diferente manera.

\section{Figura 1}

\section{Tabla 2}

En ese sentido, el presente documento busca medir el impacto de la política monetaria sobre el consumo, demostrando que en sociedades con altos niveles de heterogeneidad en los ingresos de los agentes, los mayores efectos se dan a través del canal indirecto (ingreso laboral) que por el canal directo que es la utilización misma del instrumento de política monetaria.

La estructura del documento inicia con una $1^{\circ}$ sección el cual presenta una breve introducción al tema, motivando el objetivo de la investigación; luego en la $2^{\circ}$ sección se presenta una revisión de la literatura donde se muestra el entendimiento de la relación entre consumo y el instrumento de política monetaria. En la $3^{\circ}$ sección se analiza el modelo neokeynesiano con agentes heterogéneos en una economía emergente y abierta; y su importancia en la medición del impacto del instrumento de política monetaria sobre el consumo agregado. Seguido de la $4^{\circ}$ sección donde se presenta un simple modelo de economía pequeña y cerrada que por lo pronto no asume rigideces de precios. Ello bajo el esquema de un equilibrio general entre el comportamiento de los consumidores; la empresa; política monetaria; para llegar al equilibrio de largo plazo. Por último en la $5^{\circ}$ sección se presentan algunas conclusiones.

\section{REVISIÓN DE LA LITERATURA}

En esta sección se realizará una breve revisión de aquellos trabajos que analizan cómo la política monetaria incide dentro del consumo, el mecanismo de transmisión que se utiliza para observar la efectividad de la política monetaria y si es que este genera mayor igualdad en los ingresos, que son heterogéneos y muy vulnerables a diversos choques idiosincráticos sobre todo en países emergentes.

\section{La transmisión de la politica monetaria sobre el consumo}

La decisiones de las personas entre consumir o ahorrar dependen esencialmente de la herramienta de política monetaria cuya transmisión en el tiempo sobre las variables agregadas es importante entender en parte porque, como menciona Romer (2012), hay razones fundamentales como la interacción del mercado de fondos prestables, donde interviene claramente la tasa de interés, y su efecto en los consumidores.

Inicialmente, trabajos como el de Sidrauski (1967) introducen el entendimiento de la demanda de dinero y sus efectos en la utilidad de los agentes, cuya acción se limita únicamente al problema de generarla más no responden a las razones básicas en el uso de la misma. Así, comprende que en el bienestar de las familias los agentes no solamente reciben salarios como ingresos, sino también reciben (o pagan) rentas de diferentes activos (ej. bonos y acciones, actividades de alquiler de departamentos o casas, etc.) interviniendo esencialmente la tasa de interés.

Sin embargo, una implicancia clave de la transmisión es que el gasto de las familias, asumiéndose un ingreso permanente con expectativas racionales, muestra una respuesta gradual o "suavizamiento" en su trayectoria tras las acciones de la política monetaria en el tiempo. Hall (1978) enfatiza que las familias ven incrementado su consumo como respuesta de los posibles ingresos futuros, por lo que a firma que el consumo sigue una senda estocástica o llamado paseo aleatorio. Mientras que Campbell y Deaton (1989), así como años más tarde Fuhrer (2000), demuestran que dicha respuesta gradual se debe en parte a la existencia de hábitos de consumo.

Entonces, asumiendo heterogeneidad en los ingresos, los eventos aleatorios o de incertidumbre afectan el performance del consumo en las familias. Bajo los argumentos acerca del ciclo de vida - ingreso permanente, Carrol (1997) explora cómo las familias pueden afrontar dicha incertidumbre pese a contar con altos ingresos, observando que ellas pueden ser lo suficientemente pacientes que les permita contar con una "reserva" a fin de alcanzar un nivel de crecimiento de consumo promedio igual al ingreso promedio. Dichas familias "ahorradores de reserva" tratan de generar ingresos permanentes que les permita tomar 
decisiones, por lo que la política monetaria juega un rol trascendental.

Es así que una de las formas de observar la transmisión de la política monetaria sobre el consumo es a través de la PMgC. Auclert (2018), en base a datos de ingreso y consumo de hogares en 3 períodos distintos4, estima la PMgC para Italia y EEUU, observando que las ganancias y pérdidas de capital son importantes para comprender el efecto de las tasas de interés reales sobre la $\mathrm{PMgC}$. Concluye que agentes con baja $\mathrm{PMgC}$ y poseedores de activos de largo plazo, modifican sus hojas de balance tras los efectos riqueza que se producen ante políticas monetarias contractivas. Muestra además que modificaciones en el objetivo inflación pueden crear una mejor redistribución en favor de aquellos agentes con alta $\mathrm{PMgC}$, generando un mejor efecto transmisión. Asimismo, al medir las covarianzas entre el instrumento monetario y variables agregadas, concluye que las desigualdades podrían amplificar la transmisión de la política monetaria en la sociedad.

Adicionalmente, trabajos como el de Luetticke (2008) muestra el importante efecto de la política monetaria sobre las elecciones del consumo intertemporal así como del portafolio tanto en activos líquidos e ilíquidos, característica de la heterogeneidad de los ingresos, quien asume que la efectividad de la transmisión monetaria depende no solamente de la PMgC sino también de la Propensión Marginal a Invertir (PMgI). Analiza que la redistribución de los ingresos en los agentes y el canal de Fischer de una inflación inesperada son cuantitativamente importantes5.

\section{La politica monetaria y la desigualdad de ingresos}

Entonces, ¿qué efectos genera la política monetaria en el consumo y en la desigualdad de los ingresos?. En este aspecto, Romer y Romer (2004) analizan cómo la heterogeneidad en los ingresos de las familias es afectada por las variaciones en el objetivo inflación de la Reserva

\footnotetext{
4 Encuestas construidas en base a: i) Italia: Encuesta de Ingresos y Riqueza de Hogares (SHIW en sus siglas en inglés) en el 2010 ii) EEUU: Estudio Transversal de Ingresos Dinámicos (PSID en sus siglas en inglés) desde 1999 al 2013; y la Encuesta de Casto de Consumo (CES en sus siglas en ingles) desde el 2001 Gasto de Consumo (CES en sus siglas en inges) desde el 2001 al 2002

5 El autor aplica un método llamado "grid point" desarrollado por Carroll (2006) y Hintermaier y Koeniger (2010), encontrando que cuando la economía se maneja por el lado de la demanda, el canal de Fischer amplifica los efectos agregados de los choques canal fischer a choques a gastar. En contraste, asumiendo precios flexibles, el canal a gastar. En contraste, asumiendo precios flexibles, el canal
de Fischer funciona a través de la inversión y lo lleva a una expansión después de un ajuste monetario.
}

Federal, básicamente generando cambios constantes en sus hojas de balance.

Del mismo modo, Coibion et al. (2017) tomando datos de las encuestas del gasto en consumo trimestral en EEUU (CES en sus siglas en inglés) desde 1980 al 2016, demuestra que las políticas monetarias contractivas recurrentes propician una mayor heterogeneidad tanto en los salarios laborales de las personas; su consumo; así como en el gasto total. Utilizando instrumentos convencionales de primeras diferencias en la tasa de interés y agregándole innovaciones de política monetaria, demuestra que tras recurrentes choques de políticas contractivas, se generan efectos redistributivos a lo largo de 5 años, cobrando particular importancia el hecho de la composición tan diversa que existe en la cartera de ingresos de las familias.

En cambio, Sterk y Tenreyro (2018) muestran cómo mayores operaciones de mercado abierto generan un efecto riqueza negativo elevando los incentivos por ahorrar en el mercado de fondos prestables; empujando hacia abajo la tasa de interés real; causando un incremento en la demanda laboral; y consecuentemente el empleo agregado. Así, concluyen que los incentivos por ahorrar de las familias afectan a la transmisión de la política monetaria y consecuentemente a las variables agregadas.

En tanto, Claus y Hoang (2019) demuestran cómo el incremento de la tasa de interés afecta a las expectativas de los agentes y a sus incentivos por ahorrar. Según la encuesta de Actitudes del Consumidor, Sentimientos y Expectativas en Australia (CASiE en sus siglas en inglés) desde 1993 al 2017 y aplicando un modelo de variables latentes según la metodología de Rogibon y Sack (2004), examinan que las respuestas inmediatas del consumo se alinean correctamente a sus expectativas. Mientras que a través de un modelo de retardos autorregresivos distribuidos no linealmente según la metodología de Shin et al. (2014), en el largo plazo dichas respuestas se van ajustando en el tiempo. Así, ante una política monetaria contractiva los consumidores actualizan inmediatamente sus expectativas bajo ciertas condiciones económicas como desempleo; finanzas familiares; entre otros, concluyendo que el efecto agregado se aplica de haber una mayor cantidad de agentes prestarios y si la PMgC difiere en el tiempo. 


\section{Choques idiosincráticos en los ingresos de los agentes}

Entonces, se entiende que al anclar las expectativas se evita una mayor heterogeneidad en los ingresos propiciándose un mejor efecto en el consumo. Pero ¿qué sucede al alterarse los niveles de ingresos antes posibles choques idiosincráticos en la economía? Uno de los trabajos iniciales que incorporan la heterogeneidad de los ingresos sujeto a choques idiosincráticos es Huggett (1993) quien desarrolla la medición de dichos efectos tanto bajo un modelo de agente representativo como de agentes heterogéneos en el mercado de seguros. Encuentra que al asumir un agente representativo, no explica satisfactoriamente el retorno promedio del activo ante choques, debido a que se genera una tasa libre de riesgo muy por debajo que utilizar un modelo de agentes heterogéneos.

Otro trabajo importante es el de Aiyagari (1994) quien observa diferentes implicancias que contribuyen al ahorro preventivo6. Para ello, muestra una serie de modelos con dinámicas individuales, incertidumbre y transacción de activos lo que permite trazar un camino suave del consumo en el tiempo sin alterar las variables agregadas. Además sirve como soporte para fortalecer la idea de que el comportamiento de los consumidores, la diversidad de riquezas y la estructura de los portafolios están fuertemente ligados (en varianza) al modelo de mercados completos (agente representativo), por lo que busca construir un modelo decrecimiento estándar a là Brock-Mirman (1972).

Aiyagari (1994) observa que los agentes acumulan un exceso de capital debido a que cuentan con ingresos inciertos, sugiriendo incrementar el acceso al mercado de activos que permita generar ganancias adicionales en los hogares. Señala además que el tratamiento de modelos con agentes heterogéneos contrasta con aquellos con un agente representativo cuya dinámica individual así como la incertidumbre coinciden con el nivel agregado.

6 Cabe resaltar que uno los modelos que introducen heterogeneidad son los modelos de generaciones traslapadas (OLG en sus siglas en inglés) (Samuelson, 1958; Diamond, 1965; Blanchard y Fisher, 1989; Acemoglu, 2009) que explican los impactos de la tasa de interés sobre varios sistemas como el de pensiones; seguros de salud; mercado de empleo; transiciones demográficas en el valor de los activos financieros; entre otros (Bernal et al., 2008; Maurer, 2018; De la Croix, 2012; Weil, 1989; Bergantino,1998; Acemoglu y Johnson, 2007. Takatd, 2010). Asi también bajo una estructura del ciclo de vida parsimoniosa en un modelo de equilibrio general a la Galí y Gertler (1999), se demuestra que cambios en la política monetaria pueden afectar al sector de bienes durables y al valor real de la deuda pública.
Sin embargo, Krusell y Smith (1998) muestran que utilizar modelos dinámicos de equilibrio general con un agente representativo presentan una mejor interpretación de las variables agregadas, pese a soslayar el tratamiento de microfundamentos, y cuyos resultados son tan comparables que con los mercados incompletos (agentes heterogéneos). Pese a que los resultados del modelo con un sólo agente son difíciles de defender, formulan un modelo de crecimiento estocástico con riesgos parcialmente no asegurados y con movimientos en la productividad agregada, permitiéndoles observar que ambos mercados di eren solamente en el equilibrio estocástico pero que el capital es ligeramente superior en el primero.

En esa misma línea de análisis, Huggett y Ospina (2001) establecen que si la heterogeneidad en ingresos es asumida, el ahorro agregado para la previsión es positivo, porque la tasa de interés es forzada a la baja en economías que no tienen mucha incertidumbre idiosincrática ni restricción de liquidez. Dicho argumento se sustenta en que los agentes reducen su tenencia de activos de tal forma que la restricción de liquidez es solamente para un pequeño porcentaje de la población.

\section{EL MODELO NEOKEYNESIANO CON AGENTES HETEROGÉNEOS, LA TASA DE INTERÉS Y EL CONSUMO}

Para entender la transmisión de la política monetaria sobre el consumo, el presente documento utiliza un modelo neokeynesiano con agentes heterogéneos para la cual se reseña brevemente aquellos trabajos que presentan las virtudes del modelo; y sus efectos conseguidos a través de las distintas políticas económicas ejercidas.

\section{Estructura neokeynesiana y la politica monetaria}

Uno de los trabajos que muestra una buena perspectiva de la estructura neokeynesiana respecto a la política monetaria, y con mayor precisión, la relación entre el consumo y la tasa de interés, es el de Clarida et al. (1999). Dicho documento incorpora explícitamente a través de un modelo de equilibrio general aquellas fricciones necesarias, como son la rigidez nominal en precios, para un mejor manejo de una estructura macroeconómica apropiada en la evaluación de la política monetaria.

La política monetaria que maneja el Banco Central según Clarida et al. (1999) muestra el 
compromiso creíble de reducir la inflación, siendo un fuerte supuesto que mejora su relación con el producto, produciendo ganancias de bienestar relativos al equilibrio; y generando una senda de consumo óptima en la economía. Cabe mencionar que el modelo supone firmas con competencia monopolística, donde cada una elige su precio nominal para maximizar beneficios sujetos a restricciones en la frecuencia de precios ajustados en el futuro 7 .

En esa misma línea, se desarrollan modelos donde se analizan los efectos de la tasa de interés real en la apreciación de los términos de intercambio, impactando en las exportaciones netas según la magnitud de la elasticidad sustitución entre bienes nacionales y foráneos. Así, Clarida et al. (2001) prevé que la apertura comercial complica la inflación objetivo debido a que se debe tomar en cuenta los impactos del tipo de cambio, ajustándose la tasa de interés en respuesta a las presiones inflacionarias que se generan.

Dependiendo del grado de apertura de la economía, Woodford (1999) propone que el Banco Central puede elegir entre una política monetaria óptima el cual esté bajo discreción y reoptimizándose en cada período; o bajo el compromiso de elegir una regla vinculante. Hace la salvedad que bajo una estructura de apertura de mercado, si el Banco Central ofrece no seguir con una regla comprometida, las consecuencias del mal manejo tendrán un impacto en la tasa de interés y como consecuencia en las expectativas de consumo.

Por otro lado, Clarida (2007) también provee intuiciones en la elección de una adecuada política monetaria que encara una economía abierta, proponiendo que la apertura genera efectos de "desbordamiento" del producto extranjero al nacional; que existe además un efecto positivo del crecimiento internacional a la tasa de interés interna; y que informaciones distorsionadoras afectan negativamente a la inflación pero no de la misma forma al tipo de cambio, cuyo efecto es menos directo.

\footnotetext{
7 En la medida que la fijación de precios actuales depende de las creencias acerca de las condiciones económicas futuras, una autoridad monetaria que es capaz de señalar un claro compromiso de controlar la inflación, podría encarar de una mejor manera el efecto compensatorio entre producción de mejo plazo versus inflación. Asimismo, el nombramionto de corto plazo versus inflacion. Asimismo, el nombramiento de un banquero central quien asigna un costo relativo más alto de afrontar una alta inflación reduce el sesgo inflacionario ineficiente que se obtiene bajo discreción.
}

Estudios recientes como el de Kaplan et al. (2017) también demuestran que cambios en la tasa de interés afecta el consumo de las familias, pero a diferencia de Clarida et al., este efecto es a través de canales directos e indirectos. El modelo que desarrollan muestra cómo el efecto total de la política monetaria es afectado por la presencia de heterogeneidad en los ingresos de los individuos sumados a la existencia de mercados incompletos, como lo señala también el trabajo de Auclert (2018).

Por último, el documento realizado por Menna y Tobal (2018), quienes utilizan el modelo de Cúrdia yWoodford (2016), bajo una economía abierta analizan la respuesta de la política monetaria ante distintos choques y cuáles son las consecuencias en el diferencial de tasas de interés. Sus resultados sugieren que ante la apertura de la cuenta capital y una dependencia de los flujos de capitales, disminuye la efectividad de la política monetaria8.

\section{Heterogeneidad de los ingresos y su impacto en el consumo}

¿La heterogeneidad en los ingresos mejora el impacto de las decisiones de consumo de las familias cuando hay cambios en la tasa de interés? Trabajos que tienen como enfoque de agente representativo buscan responder la relación entre los cambios en la tasa de interés respecto al consumo en el largo plazo. Por ejemplo, Campbell y Deaton (1989) utilizan diversos modelos macroeconométricos de series de tiempo bajo este enfoque pero cuyos resultados no son tan alentadores, encontrando que existe una muy baja sensibilidad entre ambas variables. Pese a ello, Krussel y Smith (1998) encuentran que utilizar agentes heterogéneos también tiene similares impactos pero en ciertas variables económicas, enfocándose para demostrarlo en el producto agregado y la respuesta de la inversión a choques de productividad9.

Entonces, ¿cuáles son las ventajas o desventajas de utilizar un modelo de agentes heterogéneos?

\footnotetext{
8 El trabajo de Fahri y Werming (2014) relacionan la política monetaria con la política fiscal, analizando como la imposición de tributos en los flujos de capitales permite mitigar depreciaciones en el tipo de cambio; incrementos en la tasa de interés; cambios en la cuenta corriente y caídas del consumo ante un estancamiento repentino de la economía.

9 Cabe resaltar que los continuos choques idiosincráticos podrian conducir a desestabilizar a las economias con serios problemas de recesión. En esa línea, Oh y Reis (2012) estudiaron que las transferencias públicas a la población ubicada en la línea de pobreza aliviaba el costo de la recesión económica, siendo el Reis (2016) observaron que el rol activo de los estabilizadores automáticos para disminuir las fluctuaciones económicas cuando la política monetaria fue muy efectivo.
} 
A partir del modelo neokeynesiano de Clarida et al. (1999) que intensifica la influencia de la política monetaria como principal mecanismo de transmisión, es que surgen trabajos que comienzan a fortalecer el enfoque de agentes heterogéneos.

As, Postel-Vinay y Robin (2002) muestran que las ventajas de utilizar un enfoque de agentes heterogéneos yace en la distribución de los ingresos que juega un rol relevante en el mercado de trabajo. Observaron que los salarios fluctúan menos que la productividad laboral el cual puede sufrir cambios significativos ante choques idiosincráticos. Así, sustenta que la heterogeneidad genera un mejor análisis en la distribución real de los ingresos y explica mejor las fluctuaciones económicas y políticas de estabilización.

Del mismo modo, Canzoneri (2007) además de Hall y Milgrom (2008), bajo el enfoque de heterogeneidad, encuentran que la tasa de interés sufre desviaciones que pueden haber afectado al consumo agregado en una medida limitada, armándose que hay evidencia empírica para explicar la fuerte respuesta del consumo agregado a movimientos de la tasa de interés reales.

\section{Modelo de dos agentes}

Otros trabajos comienzan a diversificar y comparar el enfoque de heterogeneidad en los ingresos con un modelo neokeynesiano de dos agentes (TANK en sus siglas en inglés), es decir "pobres (familias Ricardianas)" y "no pobres (familias No Ricardianas)". Galí et al. (2007) asume que éstos últimos tienen acceso al mercado de capitales y que el incremento de su consumo es la respuesta a cambios en la tasa de interés generado por los impulsos fiscales. Mientras que Bilbiie (2008) analiza, desde el lado de la política monetaria, que los "pobres" responden favorablemente a los ciclos económicos debido a que su consumo depende de la distribución de los ingresos definido por el mercado laboral.

En tanto, Sappelli y Pistaferri (2010) observan que los efectos ingreso negativos generados por la tasa de interés incrementa la inequidad en la población. Aducen que el consumo no responde a ingresos transitorios, por lo que si no se asume heterogeneidad en los ingresos se evita conocer la riqueza líquida del individuo y la composición de los efectos de hoja de balance.
Sin embargo, Werning (2015) señala que algunas veces los canales directos e indirectos se compensan de manera exacta, de modo que el efecto general de los cambios en la tasa de interés sobre el consumo no se modifica respecto a un modelo con un solo agente. Coibion et al. (2017) encuentran que la política monetaria tiene un impacto positivo pero pequeño dentro de la inequidad.

En esa línea, Bilbiie (2017) demuestra que la política monetaria es una consecuencia de los efectos indirectos. La distribución de los ingresos de la población más pobre responde más a los ciclos económicos que a los ingresos individuales. No obstante, ello sucede bajo el supuesto de choques persistentes. Entonces, la diferencia entre asegurarse con modelos con agentes heterogéneos contra los mayores márgenes de riesgo idiosincrático, es más importante cuando este proviene de la política monetaria. Es decir, demuestra que con personas de extrema pobreza, la política monetaria es más poderosa en todos los horizontes.

\section{Modelos con una distribución finita de agentes}

Pero es el trabajo de Kaplan et al. (2017) el que cobra especial significancia porque demuestran, bajo el supuesto de rigidez nominal en los precios y con una aplicación estadística rigurosa, que las familias que carecen de portafolios con activos ilíquidos muestran poca relación a los cambios de las tasas de interés, no pudiendo suavizar su consumo ante choques idiosincráticos; consumiendo su ingreso laboral y reduciendo su liquidez monetaria. El modelo también contempla choques idiosincráticos en los ingresos.

Desarrollan un análisis de las distribuciones reales de riqueza y las $\mathrm{PMgC}$ de un conjunto de familias quienes utilizan múltiples activos con diferentes grados de liquidez. Así, enmarcan el análisis comparando los dos tipos de estructuras de consumo y demostrando que el efecto total de la política monetaria sobre el consumo se trasmite a través de la elasticidad de sustitución y de la persistencia de la política.

Asumen que la tasa de descuento es igual a la tasa de interés y que en el largo plazo el PBI corriente es igual al PBI potencial. Los resultados demuestran que, con el efecto directo, cambios en la senda de la tasa de interés modificaría la trayectoria del consumo en el tiempo. Mientras 
que con el efecto indirecto, movimientos en la tasa de interés inducen a los mercados a invertir menos generando cambios en el ingreso laboral, lo que conduce a un menor consumo.

$$
d C_{0}=\underbrace{\int_{0}^{\infty} \frac{\partial C_{0}}{\partial r_{t}} d r_{t} d t}+\underbrace{\int_{0}^{\infty} \frac{\partial C_{0}}{\partial Y_{t}} d Y_{t} d t}
$$

\section{Efecto-Directo Efecto-Indirecto}

La ecuación (1) muestra los cambios que tiene el nivel de consumo ( $\mathrm{C} 0$ ); ante cambios es la tasa de interés nominal (rt); y del nivel de ingreso del consumidor (Yt), ambos medidos en el tiempo (t). Lo interesante de su artículo es que encuentran la reacción del consumo ante cambios en la tasa de interés y el ingreso comparando las dos estructuras:

- Modelo con un agente: observan que el efecto directo es cercano al $95 \%$ y el indirecto cercano al $5 \%$, es decir, muestra que la elasticidad sustitución es cuasi perfecta y que la expansión monetaria es persistente.

- Modelo con agentes heterogéneos: observan que bajo una distribución de la riqueza, el efecto directo es pequeño (33\%); mientras que bajo una distribución de la $\mathrm{PMgC}$, el efecto indirecto es grande $(66 \%)$.

Con un solo agente y contrastando con los datos, observan que existe una baja elasticidad del consumo respecto a la tasa de interés. Entonces, el mecanismo de transmisión de la política monetaria tiene mucho más significancia con el efecto indirecto debido a que empuja hacia arriba los salarios e incrementa el consumo de los individuos.

¿Cómo reaccionan estos modelos ante choques idiosincráticos? Kaplan et al. (2018) demuestran que ante diversos choques como por ejemplo a la demanda agregada, existe una fuerte equivalencia entre ambas estructuras de consumo; mientras que choques a la productividad total de factores hay una débil equivalencia; y que con choques monetarios no tienen equivalencia alguna, debido a que el mecanismo de transmisión es muy diferente.

Además analizan los impactos en la $\mathrm{PMgC}$ en los diferentes estratos socioeconómicos, encontrando que las familias con una alta restricción presupuestaria tienen una mayor $\mathrm{PMgC}$ en promedio; $\mathrm{y}$ aquellas familias con restricciones financieras tendrían problemas en el largo plazo por lo que tienden a elevar su PMgC frecuentemente10.

Por último, Kaplan y Violante (2018) encuentran que el consumo agregado bajo un modelo de agentes heterogéneos y en economías desarrolladas, depende de la dinámica del ingreso laboral y menos de la dinámica de la tasa de interés, observando que un empuje fiscal es más poderoso que una política monetaria11. Entonces, la pregunta surge inmediatamente: ¿es igual el efecto para economías emergentes y abiertas?

\section{MODELO}

Se presenta a continuación un modelo, sin rigideces de precios, con dos familias (TANK) los cuales están representados por agentes Ricardianos y agentes No Ricardianos. Según Debortoli y Galí (2017), los modelos HANK y TANK comparten una misma característica y es que en un momento determinado del tiempo una fracción de los consumidores enfrentan a una restricción de endeudamiento, lo que no permite ajustar su consumo en respuesta a variaciones de las tasa de interés o al ingreso. Si bien los modelos TANK pueden capturar una de las dimensiones de la heterogeneidad como lo es la predicción de las fluctuaciones del consumo agregado ante choques agregados, no podría abordar otros tipos de cuestiones que aborda la misma heterogeneidad.

Para desarrollar el modelo, se propone un equilibrio general dinámico estocástico (DSGE en sus siglas en inglés) con algunas fricciones para una economía con un mercado abierto. Desde el lado del consumidor, se encuentran familias Ricardianas y No Ricardianas, las cuales tienen hábitos en su consumo. Mientras que por el lado de las firmas, éstas presentan costos de ajuste y tasa de utilización del capital. Desde el lado del Estado, se considera que el gasto es exógeno por lo que no tendría ningún efecto dentro del modelo; mientras que existe una regla de Taylor que fija la política monetaria.

10 Trabajaron datos microeconómicos y mostraron que el portafolio elegido por las familias mantiene una fracción considerable de riqueza líquida (dinero), lo cual es cercana a cero; encarando un alto costo de obtener créditos dentro del mercado financiero (Kaplan, 2014).

11 Por ejemplo, una especial característica es que existe una cantidad considerable de fricciones nominales en la búsqueda de bienes de consumo en los mercados por ejemplo fricciones en el mercado de trabajo (monopolio bilateral) endogenizando los riesgos idiosincráticos de dicho mercado; o en las posiciones de portafolios donde una gran cantidad de personas están apalancadas de activos (principalmente en paison desarrincipalmente el tiempo donde las diversas hojas de balance están mucho más expuestas a movimientos de los precios de los activos que otras variables, entre otros. 


\section{Consumidores: Ricardianos y No Ricardianos}

Los consumidores optimizan tanto el consumo de bienes y servicios como su oferta de trabajo intertemporalmente; mientras que la segunda sólo optimiza su consumo a través de su ingreso laboral. Así, bajo la especificación de las preferencias con aversión relativa al riesgo constante (CRRA), se define el problema de las familias Ricardianas en la siguiente ecuación.

$V_{t}=E_{t}\left(\sum_{k=0}^{\infty} \beta^{k}\left\{\frac{\left(c_{t+k}^{R}-b c_{t+k-1}^{R}\right)^{1-\sigma}-1}{1-\sigma}-\frac{N_{t+k}^{R}{ }^{1+\varphi}}{1+\varphi}\right\}\right)$

donde la cantidad consumida agregada de un bien disponible en el mercado en el tiempo $(\mathrm{t})$ de la familia ricardiana $\left(\mathrm{C}^{\mathrm{R}}\right)$ se descuenta por un factor $\beta \in(0 ; 1)$ y por horas de tiempo trabajadas $\left(\mathrm{N}^{\mathrm{R}}\right)^{12}$. Asimismo, presentan hábitos de consumo con un costo de ajuste (b) de las costumbres o preferencias (Ferson y Constantinides, 1991) en el tiempo; una elasticidad intertemporal de sustitución del consumo $(\sigma)$ que captura los cambios en el patrón de consumo ante cambios en la tasa de interés; y la inversa de la elasticidad intertemporal de sustitución de Frisch $(\varphi)$ que mide la reasignación de las horas trabajadas frente a un cambio en el salario, manteniendo constante la utilidad marginal del ingreso.

Así, la maximización de su bienestar está sujeto a una secuencia de flujos de restricciones presupuestarias dado por:

$$
P_{t} C_{t}^{R}+D_{t}=W_{t} N_{t}^{R}+\Pi_{t}-T_{t}+\left(1+r_{t-1}\right) D_{t-1}
$$

donde hay un precio por el consumo del bien (P); los salarios nominales denotados por (W); además cuentan con acceso al mercado de capitales por lo que consumen bonos cupón cero descontados nominalmente sin riesgo y que maduran en el período $(\mathrm{t}+1)$ a una tasa de interés real $(\mathrm{r})^{13}$, pero como es una economía abierta, estos se convierten en deuda, el cual está representado por (D). Asimismo, reciben utilidades $y$ dividendos de sus firmas $(\pi)$ descontado de impuestos (T) .

Considerando que existen hábitos con formaciones externas, los agentes maximizan su utilidad tanto en el tiempo $(\mathrm{t}) \mathrm{y}(\mathrm{t}+1)$, por lo que el problema en el período $(\mathrm{t})$ es:

12 Se asume que la función de utilidad es continua y diferenciable, donde la utilidad marginal del consumo es positiva y decreciente; mientras que la desutilidad marginal del trabajo es positiva y creciente.

13 Donde la tasa de interés nominal está representada por la tasa de interés internacional así como el nivel de prima de riesgo. s.a

$$
U_{t}=\left(\frac{\left(C_{t}^{R}-b c_{t-1}^{R}\right)^{1-\sigma}-1}{1-\sigma}-\frac{N_{t}^{R^{1+\varphi}}}{1+\varphi}\right)
$$

$$
0=W_{t} N_{t}^{R}+\Pi_{t}-T_{t}+\left(1+r_{t-1}\right) D_{t-1}-C_{t}^{R}-D_{t}
$$

Maximizando a través de la función de Lagrange, se obtienen las condiciones de primer orden para el consumo $\left(\mathrm{C}^{\mathrm{R}}\right)$ y la oferta de trabajo $\left(\mathrm{N}^{\mathrm{R}}\right)$ para la familia Ricardiana.

$$
\begin{aligned}
& \frac{\partial L_{t}}{\partial C_{t}^{R}}=\left(C_{t}^{R}-b C_{t-1}^{R}\right)^{-\sigma}-\lambda_{t}=0 \\
& \frac{\partial L_{t}}{\partial N_{t}^{R}}=-N_{t}^{R \varphi}+\lambda_{t} W_{t}=0
\end{aligned}
$$

Con ambas ecuaciones se conoce un punto de inicio tanto del consumo como el de la oferta laboral en el tiempo (t). Ahora, para resolver el problema del consumidor es necesario obtener un punto final referencial del consumo $(\mathrm{t}+1)$ con el objetivo de conocer su trayectoria en el tiempo, así que debe maximizarse la función de utilidad en función a un consumo futuro (forward - looking):

$$
\begin{gathered}
U_{t+1}=\beta E_{t}\left(\frac{\left(C_{t+1}^{R}-b C_{t}^{R}\right)^{1-\sigma}-1}{1-\sigma}-\frac{N_{t+1}^{R}{ }^{1+\varphi}}{1+\varphi}\right) \\
\text { s.a } \quad 0=\beta\left(W_{t+1} N_{t+1}^{R}+\Pi_{t+1}-T_{t+1}+\left(1+r_{t}\right) D_{t}-C_{t+1}^{R}-D_{t+1}\right)
\end{gathered}
$$

bajo las condiciones de primer orden se muestra el cambio que tiene el consumo para el período $(t+1)$ en la siguiente ecuación:

$$
\frac{\partial L_{t+1}}{\partial C_{t+1}^{R}}=E_{t}\left(\left(C_{t+1}^{R}-b C_{t}^{R}\right)^{-\sigma}\right)-\lambda_{t+1}=0
$$

Así, realizando reemplazos dentro las ecuaciones que son las condiciones de primer orden en tiempo (t), se obtiene la oferta laboral óptima que ofrece el consumidor las cual está en función al consumo; el costo de ajuste de los hábitos de consumo; así como al salario nominal:

$$
N_{t}^{R \varphi}=\left(C_{t}^{R}-b C_{t-1}^{R}\right)^{-\sigma} W_{t}
$$

diferenciando respecto a la deuda se obtiene:

$$
\begin{gathered}
\frac{\partial L_{t}}{\partial D_{T}}+\frac{\partial L_{t+1}}{\partial D_{T}}=0 \\
-\lambda_{t}+\beta \lambda_{t+1}\left(1+r_{t}\right)=0
\end{gathered}
$$

Reemplazando las ecuaciones (4), (6) y (8) se obtiene la ecuación de Euler, donde la trayectoria 
del consumo está dado por el factor de descuento $(\beta)$; el operador forward looking; así como de la tasa de interés nominal como se observa en la siguiente ecuación.

$$
\frac{1}{\beta\left(1+r_{t}\right)}=E_{t}\left(\left(\frac{c_{t+1}^{R}-b c_{t}^{R}}{c_{t}^{R}-b c_{t-1}^{R}}\right)^{-\sigma}\right)
$$

Por el lado de las familias No Ricardianas, estas cuentan con una función de utilidad, que a diferencia de sus predecesores, no maximizan intertemporalmente sino que sólo consumen su nivel de ingreso laboral en un momento determinado ( $\mathrm{t}$ ). Por lo tanto, su consumo está representado por $\left(\mathrm{C}^{\mathrm{NR}}\right)$, las cuales tienen las mismas prerrogativas que los agentes Ricardianos como lo es tener un factor de descuento ( $\beta$ ); hábitos de consumo (b); horas de tiempo trabajadas $\left(\mathrm{N}^{\mathrm{NR}}\right)$; y además las elasticidades intertemporales de consumo de oferta laboral $(\sigma, \varphi)$, respectivamente como se observa en la siguiente ecuación:

$$
V_{t}=E_{t}\left(\sum_{k=0}^{\infty} \beta^{k}\left\{\frac{\left(c_{t+k}^{N R}-b c_{t+k-1}^{N R}\right)^{1-\sigma}-1}{1-\sigma}--\frac{N_{t+k}^{N R^{1+\varphi}}}{1+\varphi}\right\}\right)
$$

el cual está sujeto también a una secuencia de flujos de restricciones presupuestales según la siguiente ecuación, pero que ésta solamente se basa en un ingreso laboral:

$$
C_{t}^{N R}=W_{t} N^{N R}
$$

Así también se consideran hábitos con formaciones externas, por lo que los agentes No Ricardianos maximizan su utilidad en el tiempo $\mathrm{t}$, sin considerar su futuro:

s.a

$$
U_{t}=\left(\frac{\left(C_{t}^{N R}-b C_{t-1}^{N R}\right)^{1-\sigma}-1}{1-\sigma}-\frac{N_{t}^{N R^{1+\varphi}}}{1+\varphi}\right)
$$

$$
0=W_{t} N_{t}^{N R}-C_{t}^{N R}
$$

Por lo que optimizando a través de la función de Lagrange se obtienen las condiciones de primer orden para $\left(\mathrm{C}^{\mathrm{NR}}\right)$ y $\left(\mathrm{N}^{\mathrm{NR}}\right)$ para la familia No como se observa en las ecuaciones:

$$
\begin{aligned}
& \frac{\partial L_{t}}{\partial C_{t}^{N R}}=\left(C_{t}^{N R}-b C_{t-1}^{N R}\right)^{-\sigma}-\lambda_{t}=0 \\
& \frac{\partial L_{t}}{\partial N_{t}^{N R}}=-N_{t}^{N R^{\varphi}}+\lambda_{t} W_{t}=0
\end{aligned}
$$

y la ecuación del empleo para la familia de los No Ricardianos :

$$
N_{t}^{N R^{\varphi}}=\left(C_{t}^{N R}-b C_{t-1}^{N R}\right)^{-\sigma} W_{t}
$$

\section{Firmas}

Desde el lado de las firmas, existe un gran número de ellas idénticas que operan en la economía produciendo un bien de consumo homogéneo para el mercado en un determinado momento $(\tau)$. El problema que enfrenta es maximizar sus beneficios introduciendo para ello la función consumo que reproduce los hábitos de consumo de las familias Ricardianas; además de la elasticidad de consumo $(\sigma)$ y el factor de descuento $(\beta)$, que visto desde otra óptica, no es más que la ecuación de Euler donde se despeja el valor presente que tendría que afrontar la firma para cubrir la demanda de los consumidores; post multiplicado por la función beneficios representado por $\left(\omega_{t}\right)$

$$
\begin{gathered}
\Omega_{t}=E_{t}\left[\sum_{k=0}^{\infty} \beta^{k}\left(\frac{c_{t+k+1}^{R}-b C_{t+k}^{R}}{c_{t}^{R}-b C_{t-1}^{R}}\right)^{-\sigma} \omega_{t+k}\right] \\
\omega_{t+k}=A_{t+k}\left(K_{t+k-1} u_{t+k}\right)^{\alpha} H_{t+k}^{1-\alpha}-W_{t+k} H_{t+k}-A C\left(I_{t+k}, K_{t+k-1}\right)-I_{t+k}
\end{gathered}
$$

Las familias son dueñas del capital presentando una función de producción, donde las firmas utilizan niveles determinados de sus factores de producción como lo es el capital $\left(\mathrm{k}_{\mathrm{t}}\right)$ que además puede incrementar aún más su producción utilizando más intensamente el capital humano (pe: trabajar a dos turnos) determinado por $\left(\mathrm{u}_{\mathrm{t}}\right)^{14}$. Del mismo modo, demandan trabajo $\left(\mathrm{H}_{\mathrm{t}}\right)$; ambos pre multiplicados por sus respectivos costos de factores $\left(\mathrm{W}_{\mathrm{t}}\right) \mathrm{y}\left(\mathrm{r}_{\mathrm{t}}\right)$.

$$
Y_{t}=A_{t}\left(K_{t-1} u_{t}\right)^{\alpha} H_{t}^{1-\alpha}
$$

Además se observa una tecnología determinada por (At) donde $\left(a_{t} \equiv \log A_{t}\right)$ la cual evoluciona estocásticamente con el tiempo de acuerdo a un proceso autorregresivo de orden $\operatorname{AR}(1)$ :

$$
a_{t}=\rho a_{t-1}+\epsilon_{t}
$$

Por el lado de los costos, estos se encuentran sujeto a la ley de movimiento del capital donde la familia no solamente ofrece bienes de capital físico sino también, como se mencionó, la intensidad en el uso del capital humano denotado

14 La función tiene pendiente positiva, lo que implica que a mayor utilización del capital mayor depreciación del stock capital. Asimismo, la segunda derivada, lo que implica que dicha el stock de capital se deprecie más rápidamente de lo usual. 
por $\left(u_{\tau}\right)$, el cual representa una depreciación endógena debido a la mayor rapidez de su deterioro en el tiempo y/o posiblemente por el bajo incentivo que le dan los propietarios a dicho capital:

$$
\begin{array}{r}
K_{t}=I_{t}+\left(1-\delta\left(u_{t}\right)\right) K_{t-1} \\
\delta\left(u_{t}\right)=\delta+\frac{d}{1+z} u_{t}^{1+z}
\end{array}
$$

Es necesario precisar que la utilización intensiva del capital tiene algunos beneficios como i) expandir el producto sin presionar al mercado de trabajo, por lo que la correlación entre salario y producto disminuye; y ii) reduce la volatilidad de la inversión dado a que la demanda por capital es absorbida por la intensidad en su uso.

Asimismo, como se refirió líneas arriba, dentro de la función de costos se introducen los ajustes al capital. Básicamente menciona que dichas familias deciden separadamente el capital óptimo a utilizar y la ejecución de la inversión por lo mismo que existen dichos ajustes además de niveles determinados de (I).

$$
A C\left(I_{t}, K_{t-1}\right)=\frac{v}{2}\left(\frac{I_{t}}{K_{t-1}}-\frac{I}{K_{t}}\right)^{2} K_{t-1}
$$

La solución del sistema de ecuaciones consiste en encontrar las funciones de política o variables control, las cuales dependen de las variables de estado y/o algunas variables exógenas. En ese sentido, las familias deciden optimizar sus ingresos donde se encuentra el capital físico y humano $(\mathrm{k}, \mathrm{u})$ y la demanda de trabajo $(\mathrm{H})$; por lo que las variables control son $\mathrm{k}$; H; u. Así aplicando las condiciones de primer orden en la función de beneficios se obtiene:

$$
\begin{gathered}
\frac{\partial \Omega_{t}}{\partial u_{t}}=\alpha A_{t} K_{t-1} u_{t}^{\alpha-1} H_{t}^{1-\alpha}-\delta^{\prime}\left(u_{t}\right) K_{t-1}=0 \\
\delta^{\prime}\left(u_{t}\right) K_{t-1}=\alpha \frac{Y_{t}}{u_{t}} \\
\frac{\partial \Omega_{t}}{\partial \mathrm{H}_{t}}=A_{t}\left(K_{t-1} u_{t}\right)^{\alpha}(1-\alpha) H_{t}^{-\alpha}-W_{t}=0 \\
W_{t}=(1-\alpha) \frac{Y_{t}}{H_{t}} \\
\frac{\partial \Omega_{t}}{\partial K_{t}}=0
\end{gathered}
$$

En ese sentido, para determinar el capital, se tendrá que diferenciar el costo de ajuste (AC) respecto al capital, obteniéndose (ver apéndice 1 ).

$$
\frac{\partial A C\left(I_{t}, K_{t-1}\right)}{\partial K_{t}}-1+\beta E_{t}\left[M_{t}\left(A_{t+1} \alpha K_{t}^{\alpha-1} u_{t+1}^{\alpha} H_{t+1}^{1-\alpha}-\frac{\partial A C\left(I_{t+1}, K_{k}\right)}{\partial K_{t}}-\frac{\partial I_{t+k}}{\partial K_{t}}\right)\right]=0
$$

lo que bajos ciertos procesos algebraicos que se pueden observar en el apéndice 2, se obtiene:

$$
\begin{aligned}
& v\left(\frac{\lambda_{t}}{K_{t-1}}-\frac{1}{K_{t}}\right)+1=\beta E_{t}\left[M _ { t } \left(\alpha \frac{{\frac{Y_{t+1}}{t}}_{K_{t}}}{K_{t}}-v\left(\frac{t_{t+1}}{K_{t}}-\frac{1}{K_{t+1}}\right)\left(\frac{K_{t+1}}{K_{t}}\right)-\frac{v}{2}\left(\frac{t_{t+1}}{K_{t}}-\frac{t}{K_{t+1}}\right)^{2}-\right.\right. \\
& \left.\left.\left(1-\delta\left(u_{t+1}\right)\right)\right)\right]
\end{aligned}
$$

\section{Politica monetaria: metas de inflación}

La política monetaria implica que el Banco Central atenúe las fluctuaciones del ciclo económico para controlar la inflación. En ese sentido, dada la curva de Phillips, desde el lado de la demanda, esta se define como la inflación actual $(\pi)$ en función a las expectativas de inflación $\left(\pi^{\mathrm{e}}\right)$ descontado por el nivel de desempleo (u) premultiplicado por una elasticidad sustitución $(\alpha)^{15}$ :

$$
\pi=\pi^{e}-\alpha u
$$

Por lo tanto el Banco Central, que bajo la regla de Taylor $^{16}$, debe controlar la inflación que le permita minimizar la pérdida de bienestar de la sociedad:

$$
\operatorname{MinL}=\left\{\psi\left(\pi_{t}-\pi^{M}\right)^{2}+\lambda X^{2}\right\}+\Gamma\left\{\pi-\left(\pi^{e}+\alpha X\right)\right\}
$$

Entonces, bajo las condiciones de primer orden, se minimizan las brechas de inflación así como del producto obteniéndose a partir de estas la función de reacción de la política monetaria, la cual mide la inflación en función a la inflación meta $\left(\pi^{\mathrm{M}}\right)$ propuesta por el Banco Central descontado por la brecha producto (x) la cual está premultplicada por la importancia que se le da tanto a la inflación $(\psi)$ como al empleo $(\lambda)$.

$$
\pi=\pi^{M}-\left(\frac{\lambda}{\alpha \psi}\right) X
$$

\section{Equilibrio}

El mercado requiere que la cantidad producida de cada bien se iguale a la cantidad demanda, donde la fuente principal para consumir dichos bienes es únicamente dado por las familias. Debe señalarse que la inversión $\left(\mathrm{I}_{\mathrm{t}}\right.$ ) y el capital $\left(\mathrm{K}_{\mathrm{t}}\right)$ provienen de las familias Ricardianas. Por lo tanto, en el equilibrio se tiene que:

\footnotetext{
15 No obstante, desde el lado de la oferta agregada, la curva de Phillips está en función a las expectativas de inflación $\left(\pi^{\mathrm{e}}\right)$ sumado a una brecha del producto $(\mathrm{x})$; donde $(\mathrm{Y})$ es el Producto Bruto Interno (PBI); y el (Y*) es el PBI potencial, adicionándole también el operador de expectativa que sigue un proceso estocástico de ruido blanco

16 Donde la tasa de interés nominal (r) se ajusta uno a uno con ls expectativas de inflación $\pi^{\mathrm{e}}$ ) dada una tasa de interés real (R).
} 


$$
Y_{t}(i)=\omega C_{t}(i)^{R}+(1-\omega) C_{t}(i)^{N R}+I_{t}+G_{t}
$$

Asimismo, debe definirse que el consumo agregado así como el empleo agregado está compuesto por la ponderación en el consumo de familias Ricardianas y No Ricardianas, obteniéndose:

$$
\begin{aligned}
C_{t} & =\omega C_{t}^{R}+(1-\omega) \omega C_{t}^{N R} \\
N_{t} & =\omega N_{t}^{R}+(1-\omega) \omega N_{t}^{N R}
\end{aligned}
$$

$\mathrm{Si}$ se asume que dentro de las condiciones de equilibrio del mercado son que la familia no se endeuda al inicio $(\mathrm{D}=0)$; el mercado de trabajo se encuentra en equilibrio $(\mathrm{N}=\mathrm{H})$; que el gobierno financia todo tipo de gasto público con los ingresos por tributos $(\mathrm{T}=\mathrm{G})$; que los choques tecnológicos evolucionan en función a la ecuación del proceso autorregresivo; y además que los choques de demanda, principalmente de gasto público evolucionan también estocásticamente bajo un proceso autorregresivo de orden 1. Entonces se tiene que el sistema de ecuaciones es la que se presenta en el Cuadro 3:

\section{Tabla 3}

\section{Figura 2}

\section{CONCLUSIONES}

Según los resultados que se muestran en la Figura 2, ante un choque de productividad de 1 por ciento en el corto plazo, las funciones impulso respuesta de las variables generan un crecimiento en el producto (Y), aumentando el nivel de empleo (L) debido a que las firmas empiezan a incrementar su nivel de producción demandando más mano de obra con un incremento en los salarios (W). Desde el lado de la demanda agregada, dicho incremento en la producción desequilibra el mercado monetario; aumentando la tasa de interés (R). Asimismo, el incremento del producto aumenta el nivel de consumo agregado $(\mathrm{C})$; mientras que la inversión (I) se ajusta en el tiempo, por consecuencia del nuevo equilibrio en el mercado de fondos prestables.

De otro lado, el consumo agregado se compone por el consumo de los agentes Ricardianos $\left(\mathrm{C}^{\mathrm{R}}\right)$ y No Ricardianos $\left(\mathrm{C}^{\mathrm{NR}}\right)$, donde se observa que el incremento del producto aumenta bruscamente el consumo de los agentes No Ricardianos debido a que pueden suavizar mejor la senda del consumo tras la posibilidad acceder al mercado de fondos prestables. Asimismo, se aprecia que los consumos de ambos agentes tienen una forma tipo hump-shape debido a la existencia de hábitos de consumo.

Por lo tanto, se concluye que existe un mecanismo de diferenciación entre un modelo macroeconómico que asume agentes heterogéneos respecto a un agente representativo. Éste último no permite generar un mejor entendimiento de la desigualdad. Las políticas económicas que adopte un gobierno deben ser considerando aquellas familias que no tienen acceso al mercado de capitales y por consiguiente no puedan suavizar su consumo ante crisis económicas. Se deja como un tema de agenda conocer los impactos microeconómicos dentro de la sociedad y cuánto es el porcentaje de impacto de la tasa de interés sobre el consumo individual.

\section{REFERENCIAS}

[1] Acemoglu, Daron. 2009. Introduction to modern economic growth. Prince- ton University Press. pp. 1-1009.

[2] Auclert, Adrien. 2018. Monetary policy and the redistribution channel.

[3] American Economic Review. Vol. 9, No. 6, pp. 2333 - 2367.

[4] Aiyagari, Rao S. 1994. Uninsured idionsyncratuc risk and aggregate saving.

[5] Quarterly Journal of Economics, Vol. 109, No. 3, pp. 659-684.

[6] Blanchard, Olivier and Stanley Fischer. 1989. Lectures on macroeco- nomics. MIT Press Books. First Edition. pp. 664.

[7] Brock, William A. and Leonard J. Mirman. 1972. Optimal economic growth and uncertainty. The discounted case. Journal of Economic Theory, Vol. 4, pp. 479-513.

[8] Campbell, John and Angus Deaton. 1989. Why is Consumption so Smooth?. Review of Economic Studies, Vol.56, pp. 357-374.

[9] Canzoneri, M., R. Cumby y B. Diba. 2007. Euler equations and money market interest rates. A challenge for monetary policy models. Journal of Monetary Economics, Vol.54, pp. 1863-1881.

[10] Carroll, Christopher D. 1997. Bufferstock saving and the life cycle per- manent income hypothesis. Quarterly Journal of Economics, Vol. 112, No. 1, pp. 1-55.

[11] Carroll, Christopher D., Jiri Slacalek, Kiichi Tokuaka, Mathew 
[12] N. White. 2017. The distribution of wealth and the marginal propensity to consume. Quantitative Economics, Vol. 8, pp. 9771020.

[13] Carroll, Christopher D. 2006. The method of endogenous gridpoints for solving dynamic stochastic optimization problems. Economic Letters, Vol. 91, pp. 312-320.

[14] Clarida, Richard. 2007. Reflections on monetary policy choices in the open economy. Implications from an optimizing model. NBER International Se- minar on Macroeconomics. University of Chicago Press.

[15] Clarida, Richard, Jordi Gali and Marth Gertler. 1999. The scien- ce of monetary policy. A new keynesian perspective. Journal of Economic Literature. Vol. 37, pp. 16611707.

[16] Claus, Edda and Viet Hoang Nguyen. 2019. Monetary policy shocks from the consumer perspective. Journal of Monetary Economics. In press.

[17] Coibion, Olivier, Yuriy Gorodnichenko and Dmitri Koustas. 2017. Consumption inequality and the frequency of purchases. National Bureau of Economic Research (NBER) Working Papers 23357.

[18] Coibion, Olivier, Yuriy Gorodnichenko, Lorenz Kueng y John Sil- via. 2017. Innocent Bystanders? Monetary Policy and Inequality. National Bureau of Economic Research (NBER) Working Papers 23357.

[19] Crespo Cuaresma, Jesu's, Wolfgang Lutz and Warren Sanderson. 2014. Is the demographic dividend an education dividend?. Demography. Vol. 51, pp. 299315.

[20] Cu'rdia, Vasco and Michael Woodford. 2016. Credit frictions and op- timal monetary policy. Journal of Monetary Economics, Vol.84, pp. 30-65.

[21] De la Croix, David, Olivier Pierrard and Henry R. Sneessens. 2013. Aging and pensions in general equilibrium. Labor market imperfec- tions matter. Journal of Economic Dynamics and Control. Vol. 37, Issue 1, pp. 104-124.

[22] Debortoli, Davide and Jordi Gal'1. 2013. Monetary policy with heteroge- neous agents: Insights from TANK models. CREI Macro Lunch. Working Paper from Centre de Recerca en Economia Internacional.
[23] Diamond, Peter A. 1965. National debt in a neoclassical growth model.

[24] American Economic Review, Vol. 55, No. 5, Part 1, pp. 1126-1150.

[25] Fahri, Emmanuel and Iva'n Werning. 2013. A theory of macroprudential policies in the presence of nominal rigidities. NBER Working Paper, No. 19313, pp. 1-54

[26] Fuhrer, Jeffrey C. 2000. Habit formation in consumption and its implica- tions for monetary policy models. American Economic Review Vol. 90. No. 3, pp. 367390.

[27] Gali, Jordi and Mark Gertler. 1999. Inflation Dynamics: A Structural Econometric Analysis. Journal of Monetary Economics, Vol. 44, Issue 2, pp. 195-222.

[28] Gali J. and Olivier Blanchard. 2007. Real wage rigidities and the new keynesian model. Journal of Money, Credit and Banking, Vol. 39, No.1.

[29] Gali, Jordi. 2018. The State of New Keynesian Economics: A Partial As

[30] sessment. Journal of Economic Perspectives, Vol. 32, No.3, pp. 87-112.

[31] Hall, Robert. 1978. Stochastic Implications of the Life Cycle-Permanent Income Hypothesis. Theory and Evidence. Journal of Political Economy, Vol. 86, No.6, pp. 971987.

[32] Hintermaier, Thomas and Winfried Koeniger. 2010. The method of endogenous gridpoints with occasionally binding constraints among endoge- nous variables. Journal of Economic Dynamics and Control, Vol. 34, Issue 10, pp. 2074-2088.

[33] Huggett, Mark. 1993. The risk-free rate in heterogeneous-agent incomplete-insurance economies. Journal of Economic Dynamics and Control, Vol. 17, pp. 953-969.

[34] Huggett, Mark and Sandra Ospina. 2001. Aggregate precautionary savings: when is the third derivative irrelevant?. Journal of Monetary Economics, Vol. 48, pp. 373396.

[35] Hyunseung, Oh and Ricardo Reis. 2012. Targeted transfers and the fiscal response to the great recession. Journal of Monetary Economics. Vol. 59, Supplement 15, pp. S50-S64. 
[36] Kaplan, Greg, Benjamin Moll and Giovanni Violante. 2017. Monetary Policy According to Hank. American Economic Review. Vol. 108, No.3, pp. 697-743.

[37] Kaplan, Greg and Giovanni Violante. 2018. Microeconomic heteroge- neity and macroeconomics shocks. Journal of Economic Perspectives. Vol. 32, No. 3, pp. 167-194.

[38] Krusell, Per and Anthony Smith. 1998. Income and wealth heterogeneity in the macroeconomy. Journal of Political Economy, Vol. 106, No. 5.

[39] Luetticke, Ralph. 2018. Transmission of monetary policy with heterogeneity in household portfolios. CFM Discussion Paper Series (CFM-DP2018- 19). Centre For Macroeconomics, London School of Economics and Political Science.

[40] Maurer, Thomas. 2018. Asset pricing implications of demographic change.

[41] 24th Australasian Finance and Banking Conference 2011 Paper.

[42] McKay, Alisdair and Ricardo Reis. 2016. Optimal automatic stabilizers. National Bureau of Economic Research (NBER) Working Paper No.22359.

[43] Menna, L. y M. Tobal. 2018. Financial and price stability in emerging markets. The role of the interest rate, Bank of International Settlements Working Papers, No.717, pp. $1-76$.

[44] Motoyama, Takumi. 2018. Sustainability of public debt under physical and human capital accumulation in an OLG model. Journal of Economics. Vol. 127, Issue 1, pp. 1945.

[45] Nakamura, Emi and Jón Steinsson. 2018. High frequency identification of monetary non-neutrality. The information effect. Quarterly Journal of Economics, Vol. 133, Issue 3, pp. 12831330.
[46] Rigobon, Roberto and Brian Sack. 2004. The impact of monetary policy on asset prices. Journal of Monetary Economics, Vol. 51, Issue 8, pp. 1553- 1575.

[47] Romer, David H. 2012. Advanced Macroeconomics. Fourth Edition. McGrawHill Pub, pp. 1-738.

[48] Romer, Christina D. and David H. Romer. 2004. A new measure of monetary shocks. Derivation and implications. American Economic Review, Vol. 94, No.4, pp. 10551084.

[49] Samuelson, Paul. 1958. An exact consumption-loan model of interest with or without the social contrivance of money. Journal of Political Economy, Vol. 66, No. 6, pp. 467-482.

[50] Sánchez-Romero, Miguel; Gemma Abio; Concepcio' Patxot and

[51] Guadalupe Souto. 2018. Contribution of demography to economic growth. SERIES, Vol. 9, Issue 1, pp. 2764.

[52] Sidrauski, Miguel 1967. Rational choices and patterns of growth in a monetary economy. American Economic Review. Vol. 57, pp. 534-544.

[53] Sterk, Vincent and Silvana Tenreyro. 2018. The transmission of monetary policy through redistributions and durable purchases. Journal of Monetary Economics. Vol. 99, pp. 124-137.

[54] Woodford, Michael, 2003. Interest and Prices. Foundations of a Theory of Monetary Policy. First Edition. Princeton University Press, pp. 1-408. 


\section{APÉNDICE 1:}

$$
M_{t}=\left(\frac{C_{t+1}^{R}-b C_{t}^{R}}{C_{t}^{R}-b C_{t-1}^{R}}\right)^{-\sigma}
$$

Simplificando, se obtiene que:

$$
\frac{\partial A C\left(I_{t}, K_{t-1}\right)}{\partial K_{t}}+1=\beta E_{t}\left[M_{t}\left(\alpha \frac{Y_{t+1}}{K_{t}}-\frac{\partial A C\left(I_{t+1}, K_{k}\right)}{\partial K_{t}}-\left(1-\delta\left(u_{t+1}\right)\right)\right)\right]
$$

Luego:

$$
\begin{gathered}
\frac{\partial A C\left(I_{t}, K_{t-1}\right)}{\partial K_{t}}=\frac{\partial A C\left(I_{t}, K_{t-1}\right)}{\partial I_{t}} \frac{\partial I_{t}}{\partial K_{t}} \\
=v\left(\frac{I_{t}}{K_{t-1}}-\frac{I}{K_{t}}\right)\left(\frac{1}{K_{t-1}}\right) K_{t-1} \\
=v\left(\frac{I_{t}}{K_{t-1}}-\frac{I}{K_{t}}\right) \\
\frac{\partial A C\left(I_{t+1}, K_{t}\right)}{\partial K_{t}}=\frac{\partial}{\partial K_{t}}\left(\frac{v}{2}\left(\frac{I_{t+1}}{K_{t}}-\frac{I}{K_{t+1}}\right)^{2} K_{t}\right) \\
=v\left(\frac{I_{t+1}}{K_{t}}-\frac{I}{K_{t+1}}\right)\left(\frac{\left(\delta\left(u_{t+1}\right)-1\right) K_{t}-I_{t+1}}{K_{t}^{2}}\right) K_{t}+\frac{v}{2}\left(\frac{I_{t+1}}{K_{t}}-\frac{I}{K_{t+1}}\right)^{2} \\
=v\left(\frac{I_{t+1}}{K_{t}}-\frac{I}{K_{t+1}}\right)\left(\frac{K_{t+1}}{K_{t}}\right)+\frac{v}{2}\left(\frac{I_{t+1}}{K_{t}}-\frac{I}{K_{t+1}}\right)^{2}
\end{gathered}
$$

\section{APÉNDICE 2}

$$
\begin{aligned}
v\left(\frac{I_{t}}{K_{t-1}}-\frac{I}{K_{t}}\right) & +1 \\
& =\beta E_{t}\left[( \frac { C _ { t + 1 } ^ { R } - b C _ { t } ^ { R } } { C _ { t } ^ { R } - b C _ { t - 1 } ^ { R } } ) ^ { - \sigma } \left(\alpha \frac{Y_{t+1}}{K_{t}}-v\left(\frac{I_{t+1}}{K_{t}}-\frac{I}{K_{t+1}}\right)\left(\frac{K_{t+1}}{K_{t}}\right)\right.\right. \\
& \left.\left.-\frac{v}{2}\left(\frac{I_{t+1}}{K_{t}}-\frac{I}{K_{t+1}}\right)^{2}-\left(1-\delta-\frac{d}{1+z} u_{t+1}^{1+z}\right)\right)\right]
\end{aligned}
$$




\section{TABLAS Y FIGURAS}

TABLA 1: MUNDO: ÍNDICE DE GINI, 2004 - 2015

\begin{tabular}{|c|c|c|c|c|c|c|}
\hline \multicolumn{7}{|c|}{ Países emergentes } \\
\hline Países & Argentina & Bolivia & Brasil & Chile & Colombia & Peru' \\
\hline 2004 & 48.3 & 55.0 & 56.5 & $51.5^{*}$ & 54.8 & 49.9 \\
\hline 2009 & 43.9 & 49.2 & 53.7 & 49.0 & 54.4 & 47.0 \\
\hline 2015 & $41.4^{*}$ & 46.7 & 51.3 & 47.7 & 51.1 & 43.4 \\
\hline \multicolumn{7}{|c|}{ Países desarrollados } \\
\hline Países & Alemania & Francia & Italia & Estados Unidos & Reino Unido & China \\
\hline 2004 & 30.4 & 30.6 & 34.4 & 40.5 & 36.0 & $41.0^{*}$ \\
\hline 2009 & 30.5 & 32.7 & 33.8 & $40.4^{*}$ & 34.3 & $43.7 *$ \\
\hline 2015 & 31.7 & 32.7 & 35.4 & $41.5^{*}$ & 33.2 & 38.6 \\
\hline
\end{tabular}

[Fuente] Banco Mundial.

TABLA 2: PERÚ: PMGC VS. INGRESOS

\begin{tabular}{cccc}
\hline Población "Pobre" & $1 \circ$ Decil & $1 \circ$ Quintil & $1{ }^{\circ}$ Cuartil \\
\hline $\mathrm{PMgC}$ promedio vs. bajas $\Delta \mathrm{Y}^{*}$ & 0.71 & 0.82 & 0.52 \\
\hline $\mathrm{PMgC}$ promedio vs. altas $\Delta \mathrm{Y}^{* *}$ & 0.78 & 0.60 & 0.58 \\
\hline Poblaci'on "Rica" & $10 \circ$ Decil & $5 \circ$ Quintil & $4 \circ$ Cuartil \\
\hline $\mathrm{PMgC}$ promedio vs. bajas $\Delta \mathrm{Y}^{*}$ & 0.79 & 0.72 & 0.56 \\
\hline $\mathrm{PMgC}$ promedio vs. altas $\Delta \mathrm{Y}^{* *}$ & 0.92 & 0.83 & 0.96 \\
\hline
\end{tabular}

(*) Prom. 2008-2009 / 2013-2014 / 2017 - 2018. (**) 2008-2018.

[Fuente] INEI - Encuesta Nacional de Hogares. 
TABLA 3: SISTEMAS DE ECUACIONES PARA EL EQUILIBRIO

\begin{tabular}{|c|c|c|}
\hline Agente & Ecuación & Descripción \\
\hline \multirow{6}{*}{ Familia } & $\left(C_{t}^{R}-b C_{t-1}^{R}\right)^{-\sigma}=\beta E_{t}\left(\left(C_{t+1}^{R}-b C_{t}^{R}\right)^{-\sigma}\left(1+r_{t}\right)\right)$ & $\begin{array}{l}\text { Ecuación de Euler (Consumo de familia } \\
\text { Ricardiana) }\end{array}$ \\
\hline & $C_{t}^{N R}=W_{t} N_{t}^{N R}$ & (Consumo de familia No Ricardiana) \\
\hline & $N_{t}^{R \psi}=\left(C_{t}^{R}-b C_{t-1}^{R}\right)^{-\sigma} W_{t}$ & Oferta de Trabajo (Ricardiano) \\
\hline & $N_{t}^{N R \psi}=\left(C_{t}^{N R}-b C_{t-1}^{N R}\right)^{-\sigma} W_{t}$ & Oferta de Trabajo ( No Ricardianos) \\
\hline & $K_{t}=I_{t}+\left(1-\delta-\frac{d}{1+z} u_{t}^{1+z}\right) K_{t-1}$ & Ley de Movimiento del Capital \\
\hline & $d u_{t}^{z} K_{t-1}=\alpha \frac{Y_{t}}{u_{t}}$ & Intensidad en el Uso de Capital \\
\hline \multirow{4}{*}{ Empresas } & $Y_{t}=A_{t}\left(K_{t-1} u_{t}\right)^{\alpha} H_{t}^{1-\alpha}$ & Función de Producción \\
\hline & $W_{t}=(1-\alpha) \frac{Y_{t}}{H_{t}}$ & Demanda de Trabajo \\
\hline & $H_{t}=\omega N_{t}^{R}+(1-\omega) N_{t}^{N R}$ & Demanda de Trabajo Agregada \\
\hline & Apéndice ecuación 31 & Demanda de Capital \\
\hline Monetaria & $\pi=\pi^{M}-\left(\frac{\lambda}{\alpha \psi}\right) \chi$ & Dinámica de la Inflación \\
\hline \multirow{3}{*}{ Equilibrio } & $Y_{t}=\omega C_{t}^{R}+(1-\omega) C_{t}^{N R}+I_{t}+G_{t}$ & Equilibrio del Mercado de Bienes \\
\hline & $I_{t}=\omega I_{t}^{R}$ & Inversión Agregada \\
\hline & $K_{t}=\omega K_{t}^{R}$ & Capital Agregado \\
\hline \multirow{2}{*}{ Choque } & $\ln G_{t}=\lambda \ln G_{t-1}+v_{t}$ & Choque de demanda \\
\hline & $\ln A_{t}=\rho \ln A_{t-1}+\epsilon_{t}$ & Choque de productividad \\
\hline
\end{tabular}

[Fuente] Elaboración Propia.

FIGURA 1: PERÚ: PROPENSIÓN MARGINAL A CONSUMIR, 2008

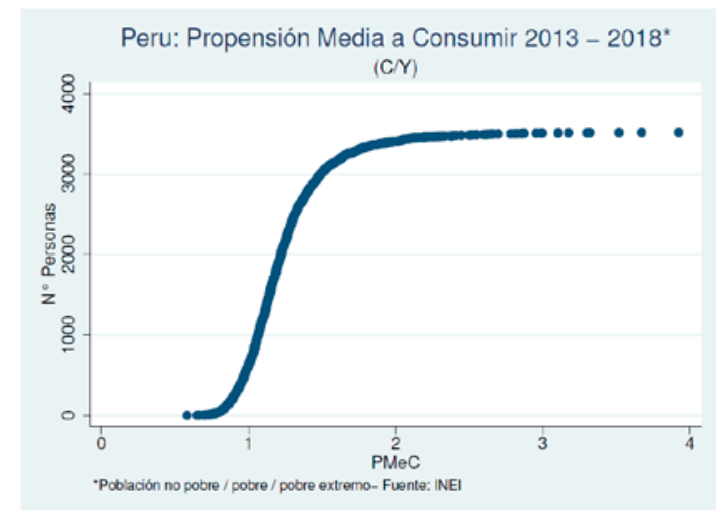


FIGURA 2: PRINCIPALES FUNCIONES RESPUESTAS

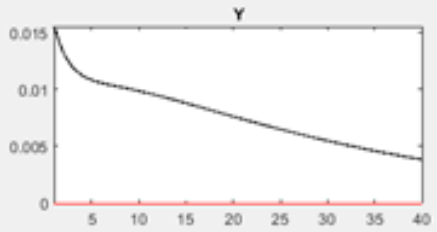

CR
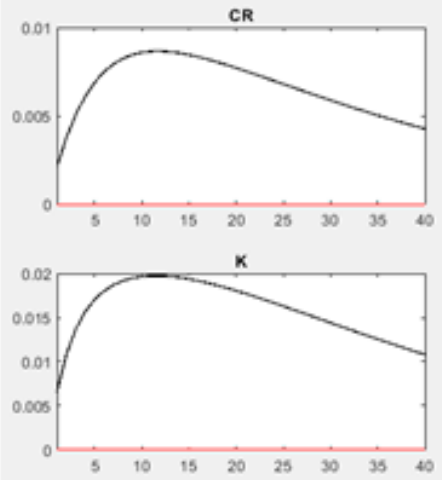

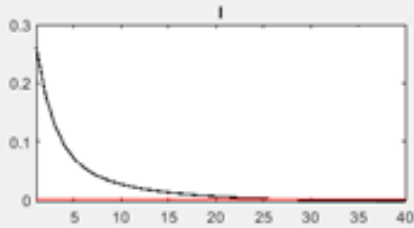

CNR
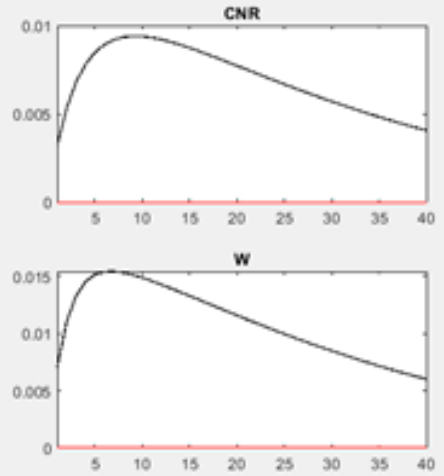
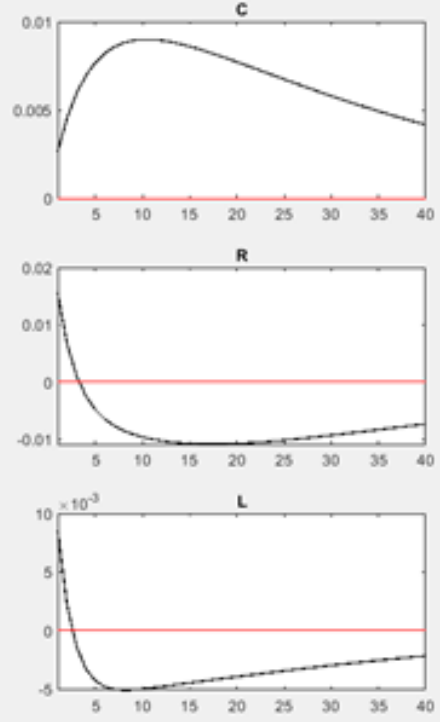